\title{
NHŨ̃NG VẤN ĐỀ CÒN TỒN TẠI TRONG VIỆC NGHIÊN CỨU QUÁ TRİNH PHỤC HỒI CẤU TRÚC CỦA THỦY TINH KIM LOẠI
}

\author{
NGUYẼ̃N THỊ NGỌC NŨ \\ Trương Đại học Công nghiệp Thành phố Hồ Chí Minh \\ nguyenthingocnu@iuh.edu.vn
}

\begin{abstract}
Bài báo viết về thủy tinh kim loại - một loại vật liệu mới với những tính chất ưu việt hứa hẹn rất nhiều ứng dụng trong tương lai. Bài báo sơ lược về các tính chất, giải thích động học thủy tinh hóa, mô tả cấu trúc và trình bày những vấn đề phức tạp còn tồn tại trong việc nghiên cứu quá trình phục hồi cấu trúc - một quá trình làm hạn chế phạm vi ứng dụng của loại vật liệu tiềm năng này.
\end{abstract}

Keywords. Thủy tinh, kim loại, thủy tinh hóa, phục hồi cấu trúc, thể tích tự do.

\section{PROBLEMS THAT EXIST IN STUDYING OF STRUCTURAL RELAXATION IN METALLIC GLASSES}

\begin{abstract}
The article presents a short review of metallic glass, the new material with superior properties that promises a lot of applications in the future. This article summarizes the properties, explains kinetics of the glass transition, describes the structure and presents complex problems that exist in studying of structural relaxation - the process that limits the scope of application of this potential material.
\end{abstract}

Keywords. Glass, metallic, glass-transition, structural relaxation, free volume.

\section{GIỚI THIỆU}

Hầu hết các kim loại kết tinh khi chúng được làm nguội từ chất lỏng thành chất rắn, quá trình sắp xếp nguyên tử của chúng thành mẫu không gian rất đều đặn gọi là ô mạng. Nhưng nếu quá trình kết tinh không xảy ra, nguyên tử thiết lập vị trí gần như rất ngẫu nhiên thì ta nhận được thủy tinh kim loại [1-3]. Vậy để tạo ra thủy tinh kim loại, kim loại phải đông đặc trước khi mạng tinh thể của chúng được tạo thành, tức là chúng phải được làm nguội với tốc độ rất cao.

Các vật liệu thủy tinh kim loại giống như kim loại ở chỗ chúng chứa các liên kết kim loại và có tính dẫn, nhưng các nguyên tử lại có cấu trúc bất trật tự như thủy tinh [4]. Do cấu trúc bất trật tự này mà thủy tinh kim loại có nhiều tính chất ưu việt hơn hẳn kim loại tinh thể, sức bền của loại vật liệu này lớn gấp mười lần polyme và giới hạn đàn hồi cao gấp hai lần các vật liệu kim loại thông thường $[5,6]$. Không những bền hơn và đàn hồi hơn nhiều so với loại thép công nghiệp tốt nhất hiện nay, thủy tinh kim loại còn thể hiện độ dẻo rất cao [7-8] . Bên cạnh đó, chúng còn rất nhẹ, hầu hết thủy tinh kim loại đều có khối lượng riêng nhỏ hơn kim loại tinh thể tương ứng [9]. Về hóa tính, thủy tinh kim loại có độ thích ứng sinh học cao và không gây dị ứng [10], có khả năng chống ăn mòn tốt. Bên cạnh những tính chất cơ học và hóa học vượt trội, thủy tinh kim loại còn có nhiều ưu điểm về từ tính như độ từ thẩm rất cao và hao hụt năng lượng thấp [11].

Với những tính chất ưu việt như đã kể trên, thủy tinh kim loại hứa hẹn rất nhiều ứng dụng trong đời sống và kỹ thuật [12-14]. Nhờ vào độ cứng và bền cao, loại vật liệu này được ứng dụng trong chế tạo vũ khí, mũi của các viên đạn xuyên áo giáp chống đạn, làm thiết bị y tế, lưỡi dao... Độ bền cao cùng với khối lượng riêng nhỏ của thủy tinh kim loại là một điều kiện lý tưởng để chúng được sử dụng vào việc chế tạo các con tàu vũ trụ trong tương lai. Các thủy tinh kim loại còn hứa hẹn cho việc sản xuất các phương tiện giao thông thân thiện với môi trường hơn. Với độ đàn hồi tốt hơn nhiều so với các kim loại tinh thể, thủy tinh kim loại có triển vọng áp dụng trong các lĩnh vực liên quan đến vật liệu nhớ hình như cơ khí chế tạo, y sinh (tim mạch, thuật chỉnh hình, thuật chỉnh răng, giải phẫu đốt sống có liên quan đến xương sống và dụng cụ phẫu thuật, nội soi,...). Nhờ vào khả năng hấp thụ và truyền năng lượng tốt do đó loại vật liệu 
này được ứng dụng trong việc sản xuất đầu gậy golf và vợt tennis...Với khả năng phân hủy tốt, thủy tinh kim loại trên cơ sở $\mathrm{Mg}$ và $\mathrm{Zn}$ có tiềm năng lớn với vai trò là vật liệu mô ghép xương phi độc tính. Sở hữu khả năng chống ăn mòn tốt, thủy tinh kim loại được ứng dụng trong công nghiệp chế tạo các thiết bị y học và thiết bị giải trí đắt tiền, linh kiện điện thoại di động, tấm phủ kháng ăn mòn cao... Nhờ vào độ từ thẩm cao và rất cứng nên thủy tinh kim loại có thể sử dụng để chế tạo đầu từ, vật liệu lưu trữ thông tin mật độ cao...

Tuy nhiên, một khó khăn gặp phải trong quá trình ứng dụng của thủy tinh kim loại là do loại vật liệu này ở trạng thái cân bằng không bền nên cấu trúc của chúng dễ dàng thay đổi đến trạng thái bền vững hơn, quá trình này được gọi là quá trình phục hồi cấu trúc (Structural Relaxation). Sự thay đổi cấu trúc này kéo theo các thay đổi về tính chất, do đó giới hạn khả năng ứng dụng của thủy tinh kim loại. Câu hỏi về cấu trúc của thủy tinh kim loại là một câu hỏi phức tạp và còn nhiều vấn đề khó hiểu. Mặc dù trên thế giới có rất nhiều nghiên cứu liên quan đến quá trình phục hồi cấu trúc, nhưng đến nay vẫn chưa có lời giải đáp thỏa đáng về cơ chế của quá trình này. Ở Việt Nam, thủy tinh kim loại là một lĩnh vực nghiên cứu hoàn toàn mói $i$. Bài báo này sẽ mô tả hiện tượng thủy tinh hóa, quá trình phục hồi cấu trúc của thủy tinh kim loại cũng như những ảnh hưởng của quá trình này đến các tính chất của chúng. Bài báo cũng sẽ khảo sát một mô hình phổ biến trong các tài liệu tham khảo về cơ chế của quá trình phục hồi cấu trúc - mô hình thể tích tự do và những vấn đề phức tạp còn tồn tại trong mô hình này.

\section{2 ĐỘNG HỌC THỦY TINH HÓA}

Thủy tinh kim loại thường được chế tạo bằng phương pháp làm nguội nhanh hợp chất nóng chảy với tốc độ cao. Động học làm nguội có thể phân tích dựa vào sự phụ thuộc của độ nhớt $\eta$ vào nhiệt độ như trên hình 1 [15]. Giả sử ban đầu hợp chất nóng chảy có nhiệt độ $T>T_{c}$, với $T_{c}$ là nhiệt độ kết tinh. Khi được làm nguội, độ nhớt của hợp chất nóng chảy bắt đầu tăng chậm (đường cong $M C$ ). Tham số quan trọng trong trường hợp này là tốc độ làm nguội $\dot{T}$. Tùy thuộc vào $\dot{T}$ mà sự thay đổi của độ nhớt có thể diễn ra theo hai khuynh hướng khác nhau. Nếu quá trình làm nguội diễn ra với tốc độ $\dot{T}$ nhỏ hơn tốc độ làm nguội tới hạn $\dot{T}_{K}$ đặc trưng cho hợp chất nóng chảy tương ứng thì ở nhiệt độ $T=T_{c}$ sẽ diễn ra quá trình kết tinh hóa, độ nhớt tăng vọt lên 10-15 lần (đường thẳng $C C^{\prime}$ ). Khi tinh thể tiếp tục được làm nguội thì độ nhớt sẽ tăng chậm đơn điệu (đường thẳng $C$ 'S). Nếu quá trình làm nguội diễn ra với tốc độ lớn hơn tốc độ làm nguội tới hạn $\left(\dot{T}>\dot{T}_{K}\right)$ thì sự kết tinh ở nhiệt độ $T=T_{c}$ không diễn ra. Hợp kim nóng chảy sẽ chuyển sang trạng thái chất lỏng siêu lạnh (chậm đông) - độ nhớt của nó tăng nhanh theo sự giảm nhiệt độ (đường cong $C E$ ). Cấu trúc của hợp chất nóng chảy chậm đông sẽ kịp thay đổi theo sự giảm nhiệt độ: mối một nhiệt độ có cấu trúc tương ứng của nó. Trạng thái này được gọi là trạng thái giả bền, một mặt thì cấu trúc của chất lỏng siêu lạnh cân bằng với nhiệt độ, mặt khác cân bằng này là không bền, bởi vì nếu dừng quá trình làm lạnh lại thì hợp chất nóng chảy sẽ kết tinh. Sự tăng nhanh của độ nhớt trong quá trình làm nguội dẫn đến sự giảm đáng kể tính linh động của nguyên tử. Khi độ nhớt đạt giá trị khoảng $10^{12} \mathrm{~Pa} . \mathrm{s}$, cấu trúc đã không kịp thay đổi theo sự giảm nhiệt độ, vật liệu sẽ đông cứng cấu hình, hay nói cách khác, vật liệu sẽ bị thủy tinh hóa. Nhiệt độ xảy ra hiện tượng này được gọi là nhiệt độ thủy tinh hóa. Điểm thủy tinh hóa trên hình 1 được kí hiệu là điểm $G_{l}$, còn nhiệt độ thủy tinh hóa $T_{g 1}$. Sự giảm nhiệt độ tiếp theo sẽ dẫn đến sự tăng tương đối chậm của độ nhớt, do vậỵ ở nhiệt độ phòng $T_{R}$ sẽ thu được thủy tinh với độ nhớt tương ứng với điểm $M_{l}$. Nếu hợp kim nóng chảy được làm nguội với tốc độ $\dot{T}_{2}<\dot{T}_{1}$ thì sự thủy tinh hóa sẽ xảy ra ở nhiệt độ $T_{g 2}<T_{g 1}$. Quá trình làm nguội xảy ra tương ứng với đường cong $M C E G_{2} M_{2}$ và ở nhiệt độ phòng sẽ nhận được thủy tinh với độ nhớt lớn hơn - tương ứng điểm $M_{2}$. Nếu tiếp tục giảm tốc độ làm nguội thì độ nhớt của thủy tinh nhận được lại tiếp tục tăng. 


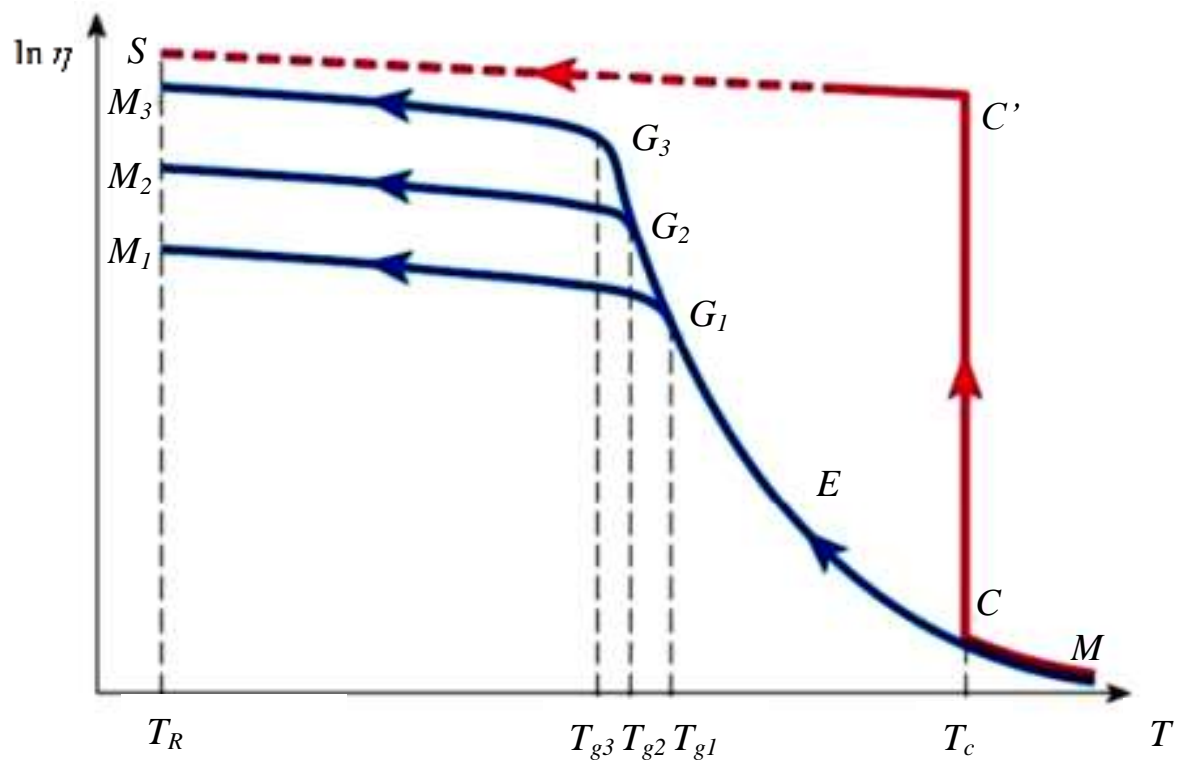

Hình 1: Minh họa động học kết tinh và thủy tinh hóa của hợp chất nóng chảy tương ứng với những tốc độ làm nguội khác nhau thông qua ví dụ về sự phụ thuộc của logarit độ nhớt vào nhiệt độ [15].

\section{QUÁ TRÌNH PHỤC HỒI CÁU TRÚC}

\subsection{Cấu trúc của thủy tinh kim loại}

Những tính chất vật lý đặc thù và độc đáo của thủy tinh kim loại được xác định bởi những đặc trưng về cấu trúc của chúng. Trên cơ sở phân tích nhiễu xạ Rơnghen đã chỉ ra rằng cấu trúc của thủy tinh kim loại tương tự cấu trúc hợp kim nóng chảy lỏng [16]. Điều này được xác nhận dựa trên kết quả thực nghiệm về một vài tính chất của thủy tinh kim loại và hợp kim nóng chảy lỏng, ví dụ như độ dẫn điện và nhiệt dung riêng của chúng có giá trị gần bằng nhau.

a

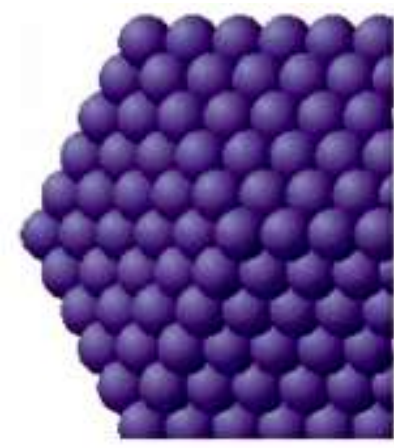

$\mathrm{b}$

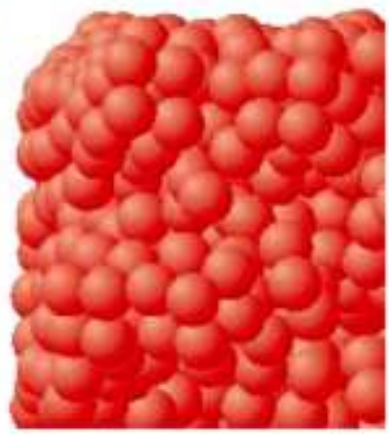

Hình 2: Mô hình trên máy tính dạng cấu trúc quả cầu rắn xếp chặt của tinh thể (a) và thủy tinh (b) [4].

Cấu trúc trật tự xa và tính tuần hoàn ở sự phân bố nguyên tử đều không tồn tại ở hợp kim lỏng và thủy tinh kim loại. Tồn tại cấu trúc trật tự gần, tức là khi xét một nguyên tử làm gốc thì bên cạnh nó với khoảng cách $d$ dọc theo một phương bất kỳ ( $d$ là bán kính nguyên tử) có thể tồn tại một nguyên tử khác nằm sát với nó, nhưng ở khoảng cách $2 d, 3 d, 4 d \ldots$ thì khả năng tồn tại của nguyên tử loại đó giảm dần. Hình 2 biểu diễn mô hình trên máy tính dạng cấu trúc quả cầu rắn xếp chặt của tinh thể và thủy tinh. Các nguyên tử được coi như những quả cầu rắn giống nhau, ở tinh thể thì chúng xếp xít nhau liên tiếp theo ba trục vuông góc, còn ở thủy tinh thì những quả cầu rắn giống như được xếp chặt trong túi cao su bó chặt một cách ngẫu nhiên tạo nên trật tự gần. Điểm khác biệt về trạng thái cấu trúc giữa thủy tinh kim loại và hợp kim lỏng tương ứng thể hiện ở cường độ dao động nhiệt của nguyên tử và tính trật tự trong sắp xếp 
nguyên tử. Ở hợp chất vô định hình rắn thì cả cường độ dao động và tính trật tự đều cao hơn ở hợp chất lỏng [17].

\subsection{Quá trình phục hồi cấu trúc}

Thủy tinh kim loại được chế tạo bằng phương pháp làm nguội nhanh hợp chất nóng chảy, do đó chúng chứa năng lượng thừa so với trạng thái tinh thể. Hệ quả là ở nhiệt độ bất kì trong thủy tinh kim loại xảy ra quá trình sắp đặt lại nguyên tử theo hướng trật tự hơn, kết quả và động lực của quá trình này là làm giảm năng lượng tự do của cấu trúc. Quá trình tự phát này là tính chất chung của tất cả các thủy tinh, và được gọi là quá trình phục hồi cấu trúc. Tính phi tinh thể vẫn được bảo toàn trong quá trình này. Tốc độ phục hồi cấu trúc, nghĩa là số lượng nguyên tử sắp đặt lại trên một đơn vị thời gian trong một đơn vị thể tích, phụ thuộc rất nhiều vào nhiệt độ và quá trình luyện nhiệt trước đó. Thông thường, ở nhiệt độ phòng và nhiệt độ thấp hơn thì tốc độ phục hồi cấu trúc không cao, mặc dù có thể nhận thấy được. Các chuyên gia đều biết rằng, những chiếc kính của sổ ở những ngôi nhà rất cổ xưa, tuổi đời hàng trăm năm thì phần dưới dày hơn một cách rõ rệt [18]. Thủy tinh giống như chảy chậm dưới tác dụng của trọng trường, sự chảy này là một biểu hiện của quá trình phục hồi cấu trúc, tuy nhiên lực hút trái đất làm cho nó có hướng.

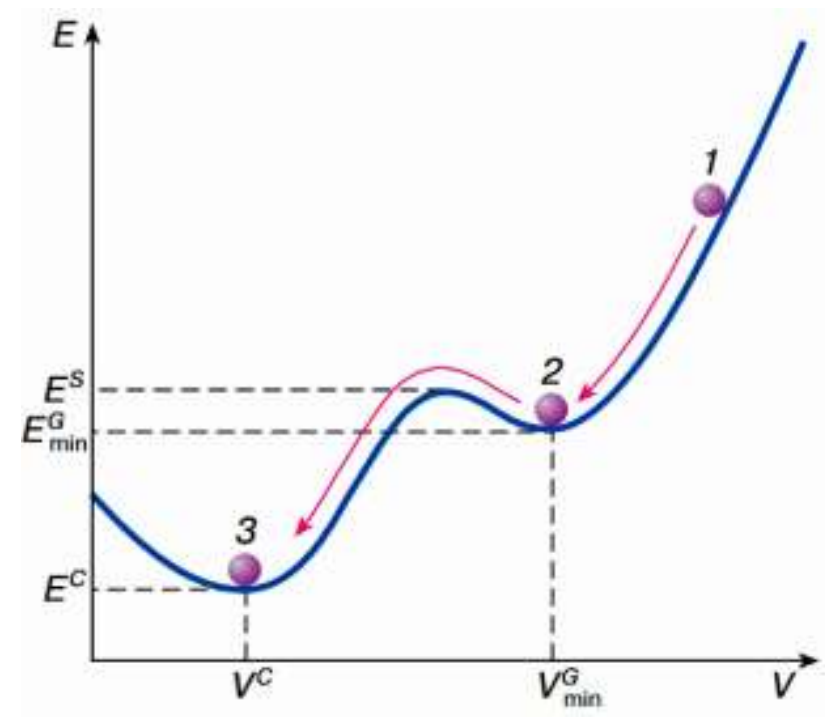

Hình 3: Minh họa động lực của quá trình phục hồi cấu trúc và tinh thể hóa [15].

Nếu được tôi luyện đủ lâu thì thủy tinh kim loại sẽ bị tinh thể hóa. Hình 3 mô tả diễn tiến chung của thủy tinh khi bị xử lý nhiệt, trên hình này chỉ ra biểu đồ sự phụ thuộc của năng lượng tự do riêng $E$ vào thể tích riêng $V$ của thủy tinh. Điểm 1 tương ứng thủy tinh ở trạng thái ban đầu với năng lượng tự do lớn nhất và thể tích riêng lớn nhất. Mọi quá trình xử lý nhiệt đều dần đến sự giảm $E$ và $V$, tức là di chuyển từ điểm 1 sang trái xuống dưới. Đây chính là quá trình phục hồi cấu trúc. Kết quả cuối cùng của nó là sự chuyển thủy tinh đến trạng thái ổn định 2 với năng lượng tự do $E_{\text {min }}^{G}$ và thể tích riêng $V_{\text {min }}^{G}$. Trong trường hợp này, nếu đưa thủy tinh ra khỏi trạng thái ổn định (di chuyển một ít sang phải hoặc sang trái so với điểm 2 , có thể làm được điều này thông qua xử lý nhiệt) thì trạng thái ổn đinh sẽ phục hồi theo thời gian. Nguyên nhân của trạng thái cân bằng ổn định ở thủy tinh hiện tại vẫn còn chưa rõ.

Thủy tinh ở trạng thái cân bằng ổn định tách khỏi trạng thái tinh thể 3 bởi rào chắn năng lượng $\Delta E=E^{S}-E_{\text {min }}^{G}$, trong đó $E^{S}$ được xác định như trên hình 3 . Nếu nhận được năng lượng $\Delta E$ từ bên ngoài thì thủy tinh sẽ bị tinh thể hóa và chuyển sang trạng thái với năng lượng tự do nhỏ nhất $E^{C}$. Sự giảm năng lượng tự do chính là động lực thúc đẩy toàn bộ quá trình vận động của thủy tinh kim loại từ trạng thái ban đầu cho đến khi bị tinh thể hóa. 


\section{3 Ảnh hưởng của quá trình phục hồi cấu trúc lên tính chất của thủy tinh kim loại}

Quá trình phục hồi cấu trúc dẫn đến những thay đổi về sự phân bố của nguyên tử trong không gian và xây dựng lại những liên kết hóa học, từ đó dẫn đến sự thay đổi của hầu hết các tính chất của thủy tinh kim loại: hệ số khuếch tán, độ nhớt, tính dẻo, dị hướng từ...[19]. Trong trường hợp này, một số tính chất thay đổi tương đối yếu, còn một số tính chất thay đổi rất mạnh. Ví dụ, khối lượng riêng của thủy tinh kim loại sau quá trình phục hồi cấu trúc có thể tăng $0,5-1 \%$, còn độ nhớt ở cùng một nhiệt độ tăng lên hàng nghìn lần [20]. Sự phục hồi cấu trúc làm cho các mô đun đàn hồi tăng lên: Mô đun Young E tăng lên 7-18\%, Mô đun cắt $\mathrm{G}$ - khoảng $8-22 \%$, Mô đun khối $(\mathrm{K})-1-8 \%$. Các nhiệt độ đặc trưng cũng bị thay đổi: nhiệt độ Debye tăng khoảng $8 \%$, nhiệt độ Curie thay đổi hơn $40 \mathrm{~K}$. Quá trình phục hồi cấu trúc, tùy thuộc vào thành phần hóa học của thủy tinh, có thể dẫn đến sự tăng hoặc giảm điện trở. Ngoài ra nó còn làm giảm lực nội ma sát, tốc độ phục hồi ứng suất, tốc độ biến dạng trượt...

Tốc độ của quá trình phục hồi cấu trúc là một tham số quan trọng đặc trưng cho sự ổn định tính chất của thủy tinh kim loại. Tốc độ này phụ thuộc rất lớn vào lịch sử luyện nhiệt của thủy tỉnh kim loại. Tính toán chính xác động học phục hồi cấu trúc dựa vào lịch sử luyện nhiệt rất phức tạp, tuy nhiên, ở dạng đơn giản hóa hình ảnh có dạng sau [21]: tốc độ phục hồi cấu trúc nhỏ ở tất cả các nhiệt độ $T<T_{a}$, trong đó $T_{a}$ là nhiệt độ luyện nhiệt lớn nhất trước đó. Vì vậy sự thay đổi tính chất của thủy tinh kim loại ở những nhiệt độ này chỉ biểu hiện sau khoảng thời gian dài. Đun nóng đến nhiệt độ $T_{1}$, với $T_{1}$ lớn hơn $T_{a}$ khoảng 10 $\mathrm{K}$ có thể tăng tốc độ phục hồi cấu trúc lên hàng nghìn, hàng triệu lần. Luyện nhiệt ở nhiệt độ $T=T_{1}$ dẫn đến sự giảm tốc độ phục hồi cấu trúc, do đó ở tất cả các nhiệt độ $T<T_{1}$ tốc độ này rất nhỏ. Nếu như tiếp tục đun thủy tinh kim loại đến nhiệt độ $T_{2}>T_{1}$ thì sự tiến triển như đã kể trên của tốc độ phục hồi cấu trúc được lặp lại.

Mặc dù có rất nhiều nghiên cứu liên quan, tuy nhiên quá trình phục hồi cấu trúc vẫn còn nhiều vấn đề chưa sáng tỏ, và cơ chế của nó vẫn còn chưa giải thích được. Dưới đây chúng ta sẽ tìm hiểu một mô hình phổ biến trong các tài liệu tham khảo về cơ chế của quá trình phục hồi cấu trúc ở thủy tinh kim loại - mô hình thể tích tự do.

\subsection{Mô hình thể tích tự do}

Mô hình thể tích tự do về cơ bản xây dựng trên bốn giả thuyết sau [22]: (1) mỗi phân tử được gắn với một thể tích cục bộ $V ;(2)$ khi $V$ tăng và đạt giá trị tới hạn $V_{c}$ thì hình thành thể tích thừa, được gọi là thể tích tự do; (3) sự dịch chuyển phân tử xảy ra khi thể tích thừa gần bằng thể tích phân tử $V_{m} ;$; (4) để phân bố lại thể tích tự do cần phải có năng lượng tự do không cục bộ. Ở mô hình này, sự thủy tinh hóa xảy ra khi mật độ thể tích tự do giảm đến giá trị tới hạn. Mật độ thể tích tự do chính là tham số giúp phân biệt giữa thủy tinh với chất lỏng và kiểm soát quá trình phục hồi cấu trúc của thủy tinh. Các tâm phục hồi gắn với thể tích tự do được hiểu như tập hợp các vùng có khối lượng riêng thấp hơn bình thường. Thủy tinh kim loại mới chế tạo đều chứa một số lượng thể tích tự do nhất định, trong quá trình luyện nhiệt những thể tích tự do này dần biến mất dẫn đến sự tăng độ nhớt và khối lượng riêng của thủy tinh kim loại. Vấn đề giải thích quá trình phục hồi cấu trúc chính là đi tìm sự phụ thuộc của mật độ thể tích tự do vào nhiệt độ. Mật độ thể tích tự do chính là đại lượng xác định động học phục hồi tính chất vật lý của thủy tinh kim loại.

Do tính bất trật tự của nguyên tử nên thể tích tự do phân bố không đồng đều trong cấu trúc vô định hình. Năm 1959 Cohen và Turnbull [23] nhận được hàm số phân bố của thể tích tự do trong cấu trúc của thủy tinh:

$$
P(\mathrm{v}) d \mathrm{v}=\frac{g}{\mathrm{v}_{f}} \exp \left(-\frac{g \mathrm{v}}{\mathrm{v}_{f}}\right) d \mathrm{v}
$$

Với $P(\mathrm{v}) d \mathrm{v}$ là xác suất thể tích trống của nguyên tử có giá trị từ $\mathrm{v}$ đến $\mathrm{v}+d \mathrm{v}, g-$ thừa số hình học

$(0.5<g<1), \mathrm{v}_{f}$ - giá trị trung bình của thể tích tự do. Sau đó Spaepen [24] và Argon [25-26] đã sử dụng phân bố này để giải thích về sự chảy dẻo của thủy tinh kim loại. Spaepen nhận được công thức biểu diễn độ nhớt $\eta$ như sau: 


$$
\eta=\frac{k T}{\nu \Omega} \exp \left(\frac{g \mathrm{v}^{*}}{\mathrm{v}_{f}}\right) \exp \left(\frac{\Delta G^{m}}{k T}\right)
$$

Trong đó $\mathrm{v}^{*}$ - thể tích cục bộ tới hạn để thực hiện một động tác cơ bản nhằm xây dựng lại cấu trúc, $\Delta G^{m}$ - năng lượng hoạt hóa của động tác này, $\Omega$ thể tích nguyên tử, $k$ - Hằng số Boltzmann, $T$ - nhiệt độ tuyệt đối. Phương trình có dạng tương tự cũng được Argon tìm ra đối với độ nhớt của thủy tinh kim loại khi khảo sát sự chảy dẻo. Trên cơ sở mô hình thể tích tự do Argon cũng đã tìm ra ranh giới gần đúng giữa sự chảy dẻo đồng nhất và không đồng nhất. Lợi thế của mô hình thể tích tự do là giúp mô tả hai dạng chảy dẻo với cùng một quan điểm. Tuy nhiên để giải thích sự phụ thuộc của độ nhớt vào thời gian nó đòi hỏi điều chỉnh 7 tham số cho phù hợp [27], điều này rõ ràng làm giảm giá trị của kết quả thu được.

Mô hình thể tích tự do tồn tại hàng loạt các vấn đề phức tạp, một trong những vấn đề đó là việc chỉ có một năng lượng hoạt hóa duy nhất, điều này thực chất không phù hợp với nhiều kết quả thực nghiệm [2831]. Vấn đề tiểp theo là câu hỏi làm thế nào để xác định chính xác thể tích tự do bằng phương pháp nhiệt động lực học. Thể tích tự do rõ ràng gắn liền với khối lượng riêng, tuy nhiên, nhiều nghiên cứu chỉ ra rằng, thời gian phục hồi thường không chỉ phụ thuộc vào khối lượng riêng [32]. Các tác giả bài báo [33] đã thực hiện phép đo khối lượng riêng của các mẫu thủy tinh kim loại $\mathrm{Pd}_{40} \mathrm{Cu}_{30} \mathrm{Ni}_{10} \mathrm{P}_{20}$ dạng mảnh và dạng khối, đồng thời tính toán tỉ số độ nhớt của chúng theo mô hình thể tích tự do. Theo kết quả tính toán, độ nhớt của thủy tinh kim loại dạng mảnh phải nhỏ hơn $10^{7}$ lần so với dạng khối. Tuy nhiên kết quả thực nghiệm cho thấy, độ nhớt của thủy tinh kim loại $\mathrm{Pd}_{40} \mathrm{Cu}_{30} \mathrm{Ni}_{10} \mathrm{P}_{20}$ dạng mảnh và dạng khối thực tế bằng nhau! Ngoài ra, có rất nhiều thực nghiệm đặt nghi vấn lớn về liên hệ giữa tâm phục hồi và thể tích trống. Ví dụ, đối với những thủy tinh kim loại cùng thành phần hóa học nhưng có sự khác biệt lớn về khối lượng riêng thì cường độ của những quá trình phục hồi cấu trúc hầu như bằng nhau [34-40]. Bên cạnh đó, các tác giả bài báo [41] đã chỉ ra rằng hợp kim $\mathrm{Pd}_{40} \mathrm{Ni}_{40-\mathrm{x}} \mathrm{Cu}_{\mathrm{x}} \mathrm{P}_{20}$ với $\mathrm{x}>30$ ở trạng thái thủy tinh có khối lượng riêng thậm chí lớn hơn ở trạng thái tinh thể. Tuy nhiên tất cả những thủy tinh kim loại này đều thể hiện tính chảy dẻo mạnh ở nhiệt độ lớn hơn và nhỏ hơn $T_{g}$. Những kết quả thực nghiệm đã kể trên cho thấy, quá trình phục hồi cấu trúc là quá trình đa diện, còn sự tiêu hủy thể tích tự do không phải là yếu tố xác định trong quá trình phục hồi cấu trúc, mà chỉ là một trong những thành phần của nó.

Quá trình phục hồi cấu trúc thường được cho là quá trình bất thuận nghịch, dẫn đến sự phục hồi bất thuận nghịch của tính chất. Nhiệt độ và thời gian luyện nhiệt càng cao thì tính chất thay đổi càng nhiều. Quá trình phục hồi cấu trúc là một trong những nguyên nhân làm hạn chế khả năng ứng dụng trong thực tiễn của thủy tinh kim loại. Tuy nhiên, có một vài thực nghiệm nói đến khả năng khôi phục hàng loạt các tính chất của những thủy tinh kim loại đã hoàn toàn phục hồi cấu trúc bằng những phương pháp luyện nhiệt chuyên biệt [42-49]. Cơ chế của sự khôi phục tính chất bằng phương pháp luyện nhiệt vẫn chưa được giải thích rõ ràng. Điều này càng làm tăng thêm tính hấp dẫn của loại vật việu này, bởi vì phạm vi ứng dụng của thủy tinh kim loại có khả năng được mở rộng.

\section{KẾT LUẬN}

Thủy tinh kim loại tuy ở thể rắn nhưng lại mang cấu trúc vô định hình như chất lỏng. Để chế tạo thủy tinh kim loại thì hợp kim nóng chảy phải được làm nguội với tốc độ rất cao. Loại vật liệu này không những bền, có độ cứng cao và tính đàn hồi tốt hơn nhiều so với kim loại thông thường mà còn có nhiều ưu điểm về hóa tính và từ tính. Với những đặc tính vượt trội, thủy tinh kim loại được coi là vật liệu tiềm năng trong tương lai và đang thu hút sự quan tâm đặc biệt của giới khoa học kỹ thuật trên thế giới.

Những ưu thế về tính chất của thủy tinh kim loại được hình thành từ những đặc điểm cấu trúc của chúng. Tuy nhiên, do trạng thái cân bằng không bền nển ở thủy tinh kim loại luôn diễ் ra quá trình phục hồi câu trúc, tốc độ của quá trình này tăng theo nhiệt độ, điều này làm hạn chế phạm vi ứng dụng của thủy tinh kim loại. Cơ chế của quá trình phục hồi cấu trúc thường được giải thích theo mô hình thể tích tự do, tuy nhiên mô hình này đang gặp rất nhiều mâu thuẫn với thực nghiệm. Ngoài ra, một số nghiên cứu phát hiện tính chất của thủy tinh kim loại sau khi phục hồi cấu trúc có thể được khôi phục, điểu này hứa hẹn khả năng mở rộng phạm vi ứng dụng của chúng. Những kết quả nhận được giúp mở ra nhiều hướng nghiên cứu liên quan đến loại vật liệu tiềm năng này. 


\section{REFERENCES}

[1] J. Langer, The mysterious glass transition, Physics Today, vol. 60, pp. 8-9, 2007.

[2] P.G. Debenedetti, F.N. Stillinger, Supercooled liquids and the glass transition, Nature, Vol. 410, pp. 259-267, 2001.

[3] А.И. Черноуцан, Физические свойства процесса стеклования, Соросовский образовательный журнал, №3., Т. 7, С. 103-109, 2001.

[4] Zolotukhin, Аморфные Металлические Материалы, Соросовский образовательный журнал. Т. 4, , С. 73$78,1997$.

[5] J.F. Loffler, Bulk metallic glasses, Intermetallics, Vol. 11, pp. 529-540, 2003.

[6] M.Telford, The case for bulk metallic glass, Applications Feature, Vol. 7, pp. 36-43, 2004.

[7] M.F. Ashby, A.L. Greer . Metallic glasses as structural materials, Scripta Materialia, Vol. 54, pp. 321-326, 2006.

[8] Yang Shao, Guannan Yang, and Kefu Yao, Nanocrystalline Phase Formation inside Shear Bands of Pd-Cu-Si Metallic Glass, Advances in Materials Science and Engineering , 2014.

[9] A. Inoue, Bulk Amorphous Alloys. Preparation and Fundamental Characteristics, vol. 4, Materials Science Foundation, Transtech, Zurich, 1999.

[10] Kazuhiro Imai, n Vivo Investigation of Zr-Based Bulk Metallic Glasses Sub-Periosteally Implanted on the Bone Surface, Journal of Materials Science and Chemical Engineering, Vol. 4, pp. 46-51, 2016.

[11] Chakri et al., Crystallization Kinetics and Magnetic Properties of $\mathrm{Fe}_{40} \mathrm{Ni}_{40} \mathrm{~B}_{20}$ Bulk Metallic Glass, Advances in Chemical Engineering and Science, Vol. 4, , pp. 36-38, 2014.

[12] Nguyễn Thị Ngọc Nữ, Trần Văn Lượng, Thủy tinh kim loại: phương pháp chế tạo và tiềm năng ứng dụng, Tạp Chí Khoa Học Trường Đại Học Cần Thơ, Số 47a, tr. 40-46, 2016.

[13] A.I. Salimon, M.F. Ashby, Y. Bréchet, A.L. Greer. Bulk metallic glasses: what are they good for? Materials Science and Engineering A, Vol. 375, pp. 385-388, 2004.

[14] Nguyen Thi Ngoc Nu and Tran Van Luong, Potential Applications of Metallic Glasses, International Journal of Science, Environme and Technology, Vol. 5 No. 4, 2016.

[15] V. A. Khonik , Стекла: структура и структурные превращения. Соросовский образовательный журнал. Т. 7, C. $95-102,2001$.

[16] Т. Эгами, Изучение структуры с помощью рентгеновской дифракции с дисперсией по энергии, Металлические стекла. Ионная структура, электронный перенос и кристаллизация. Под ред. Г. Гюнтеродта и Г. Бека, Москва: Мир, 1983.

[17] И.С. Мирошниченко. Закалка из жидкого состояния, Москва: Металлургия., 1982.

[18] Б.Н. Бушманов , Ю.А. Хромов, Физика Твердого тела, Москва: Высшая школа, 1971.

[19] Х.С. Чен, Структурная релаксация в металлических стеклах, Аморфные металлические сплавы: Сборник трудов. Под ред. Ф.Е. Люборского. Москва, 1987.

[20] A.I. Taub, Isoconfigurational flow of amorphous $\mathrm{Pd}_{82} \mathrm{Si}_{18}$, Scripta Metallurgica, Vol. 13, pp. 195-198, 1979.

[21] V.A. Khonik, Internal friction of Metallic Glass, Mechanisms and conditions of their realization, J. Phys. IV. Vol. 6, pp 591- 600, 1996.

[22] H.C. Cohen, G.S Grest, Liquid-glass transition, a free-volume approach, Physical Review B., Vol. 20, N 3, pp. 1077-1098, 1979. 
[23] M.N. Cohen, D. Turnbull, Molecular transport in liquids and glasses, Journal of Chemical Physics, 1959 , Vol. 31, pp. 1164-1169.

[24] F. Spaepen, A microscopic mechanism for steady state inhomogeneous flow in metallic glasses, Acta Metallurgica, Vol. 25, pp. 407-415, 1977.

[25] A.S. Argon, H.Y. Kuo, Free energy spectra of inelastic deformation of five metallic glass alloys, Journal of Non-Crystalline Solids, Vol. 37, pp. 241-266, 1980.

[26] A.S. Argon, Plastic deformation in metallic glasses, Acta Metallurgica, Vol. 27, pp. 47-58, 1979.

[27] K. Russew, B.J. Zappel, F. Sommer, Nonisothermal viscous flow behaviour of Pd-Ni-P glassy alloy considered as a free volume related phenomenon, Scripta Metallurgica Materialia, Vol. 32, pp. 271-276, 1995.

[28] J.A. Leake, E. Woldt, J.E. Evetts, Gaussian activation energy spectra in reversible and irreversible structural relaxation, Material Science and Engineering, Vol. 97, pp. 469-472, 1988.

[29] M.R.J.Gibbs, J.E. Evets, J.A. Leake, Activation energy spectra and relaxation in amorphous materials, Journal of Material Science, Vol. 18, pp. 278-288,1983.

[30] R. Cost, Nonlinear regression least-squares method for determining relaxation time spectra for processes with first-order kinetics, Journal of Applied Physics, Vol. 54, pp. 2137-2146, 1983

[31] P. Kruger, L. Kempen, H. Neuhauser, Determination of the effective attempt frequency of irreversible structural relaxation processes in amorphous alloys by an isothermal measurements, Physica Status Solidi, Vol. 131, pp. 391402, 1992.

[32] J.C. Dyre, The glass transition and elastic model of glass-forming liquids, Reviews of Modern Physics, Vol. 78, pp. 953-972, 2006.

[33] O.P. Bobrov, V.A. Khonik, S.A. Lyakhov, K. Csach, K. Kitagawa, H. Neuhäuser, Shear viscosity of bulk and ribbon glassy $\mathrm{Pd}_{40} \mathrm{Cu}_{30} \mathrm{Ni}_{10} \mathrm{P}_{20}$ well below and near the glass transition, Journal of Applied Physics, Vol. 100, p. 033518-1-033518-9, 2006.

[34] U. Harms, O. Jin, Schwarz R.B. Effects of plastic deformation on the elastic modulus and density of bulk amorphous $\mathrm{Pd}_{40} \mathrm{Ni}_{10} \mathrm{Cu}_{30} \mathrm{P}_{20}$, Journal of Non-Crystalline Solids, Vol. 317, pp. 200-205, 2003.

[35] A.R. Yavari , A.L. Moulec , A. Inoue , N. Nishiyama , N. Lupu , E. Matsubara, W.J. Botta , G. Vaughan, M.D. Michiel, A. Kvick. Excess free volume in metallic glasses measured by X-ray diffraction, Acta Materialia, Vol. 53, pp. 1611-1619, 2005.

[36] A.E. Berlev, O.P. Bobrov , V.A. Khonik , K. Csach, A. Juríková ,J. Miškuf, H. Neuhäuser, M.Yu. Yazvitsky Viscosity of bulk and ribbon Zr-based metallic glasses well below and in the vicinity of Tg: a comparative study, Physical Review B, Vol. 68, pp. 132203, 2003.

[37] Csach K., Bobrov O.P., Khonik V.A., Lyakhov S.A., Kitagawa K. Relationship between the shear viscosity and heating rate of metallic glasses below Tg, Physical Review B, Vol. 73, pp. 092107, 2006.

[38] S.V. Khonik, V.V. Sviridov, O.P.Bobrov, M.Yu.Yazvitsky, V.A. Khonik .Structural relaxation and recovery of bulk and ribbon glassy $\mathrm{Pd}_{40} \mathrm{Cu}_{30} \mathrm{Ni}_{10} \mathrm{P}_{20}$ monitored by measurements of infralow-frequency friction. Journal of Physics: Condensed Matter, Vol. 20, pp. 165204, 2008.

[39] Хоник С.В., Свиридов В.В., Кобелев Н.П., Язвицкий М.Ю., Хоник В.А. Кинетика структурной релаксации стекла $\mathrm{Pd}_{40} \mathrm{Cu}_{30} \mathrm{Ni}_{10} \mathrm{P}_{20}$ в объемном и ленточном состояниях по данным измерений электрического сопротивления, Физика твердого тела.,Т. 49, вып. 8, С. 1345-1351, 2007. 
[40] S.V. Khonik, L.D. Kaverin, N.P. Kobelev, N.T.N. Nguyen, A.V. Lysenko, M.Yu. Yazvitsky ,V.A. Khonik, The kinetics of structural relaxation of bulk and ribbon glassy $\mathrm{Pd}_{40} \mathrm{Cu}_{30} \mathrm{Ni}_{10} \mathrm{P}_{20}$ monitored by resistance and density measurements, Journal of Non-Crystalline Solids, Vol. 354, pp. 3896-3902, 2008.

[41] T.D. Shen, U. Harms ,R.B. Schwarz, Correlation between the volume change during crystallization and the thermal stability of supercooled liquids, Applied Physics Letter, Vol. 83, N 22, pp. 4512-4514, 2003.

[42] Н.П. Кобелев, Е.Л. Колыванов, В.А. Хоник. Влияние деформационной и термической обработок на затухание и модуль сдвига в объемном механическом стекле $\mathrm{Zr}-\mathrm{Cu}-\mathrm{Ni}-\mathrm{Al}-\mathrm{Ti}$, Физика Твердого тела, Т. 47, с. 646-649, 2005.

[43] O.P. Bobrov, K. Csach, S.V. Khonik, K. Kitagawa, S.A. Lyakhov, M.Yu. Yazvitsky, V.A. Khonik, The recovery of structural relaxation-induced viscoelastic creep strain in bulk and ribbon $\mathrm{Pd}_{40} \mathrm{Cu}_{30} \mathrm{Ni}_{10} \mathrm{P}_{20}$ glass, Scripta Materialia, Vol. 56, pp. 29-32, 2007.

[44] V. A. Khonik, Yu. P. Mitrofanov, S. A. Lyakhov, D. A. Khoviv, and R. A. Konchakov, Recovery of structural relaxation in aged metallic glass as determined by high-precision in situ shear modulus measurements, Journal of Applied Physics, Vol. 105, pp. 123521, 2009.

[45] V. A. Khonik, A. V. Lysenko, The recovery of the shear viscosity of thermally aged bulk and ribbon glassy $\mathrm{Pd}_{40} \mathrm{Cu}_{30} \mathrm{Ni}_{10} \mathrm{P}_{20}$ by rapid quenching from the supercooled liquid state, Phys. Status Solidi RRL, Vol.3, No. 2, pp. 37-39, 2009.

[46] V.A. Khonik, N.T.N. Nguyen, S.V. Khonik, N.A. Divakova, Recovery of the ability to shear stress relaxation of thermally aged bulk and ribbon glassy $\mathrm{Pd}_{40} \mathrm{Cu}_{30} \mathrm{Ni}_{10} \mathrm{P}_{20}$, Scripta Materialia, Vol. 61, pp..153-156, 2009.

[47] V.A. Khonik, N.T.N. Nguyen, S.V. Khonik , A.V. Lysenko, D.A. Khoviv, Usual stress relaxation in an 'unusual' $\mathrm{Pd}_{40} \mathrm{Cu}_{40} \mathrm{P}_{20}$ metallic glass. Journal of Non-Crystalline Solids, Vol. 355, pp. 2175-2178, 2009.

[48] N.T.N. Nguyen, S. V. Khonika, M. Yu. Yazvitski, V. A. Khonika, Recovery of the Deformability of the Aged Metallic Glass $\mathrm{Pd}_{40} \mathrm{Cu}_{30} \mathrm{Ni}_{10} \mathrm{P}_{20}$ under Conditions of Testing for Shear Stress Relaxation, Physics of the Solid State, Vol. 51, No. 3, pp. 514-517, 2009.

[49] N.T.N. Nguyen, S.V. Khonik, V.A. Khonik, Isochronal shear stress relaxation and recovery of bulk and ribbon glassy $\mathrm{Pd}_{40} \mathrm{Cu}_{30} \mathrm{Ni}_{10} \mathrm{P}_{20}$, Phys. Status Solidi A., Vol. 206, pp. 1440-1446, 2009.

Ngày nhận bài: 20/12/2017

Ngày chấp nhận đăng:22/03/2018 